\title{
Improving the Demulsification Process of Heavy Crude Oil Emulsion through Blending with Diluent
}

\author{
K. K. Salam, A. O. Alade, A. O. Arinkoola, and A. Opawale \\ Petroleum Engineering Unit, Department of Chemical Engineering, Ladoke Akintola University of Technology (LAUTECH), \\ PMB 4000, Ogbomoso, Nigeria
}

Correspondence should be addressed to K. K. Salam; kaykaysalam@yahoo.co.uk

Received 7 January 2013; Accepted 3 April 2013

Academic Editor: Andrea Franzetti

Copyright ( 92013 K. K. Salam et al. This is an open access article distributed under the Creative Commons Attribution License, which permits unrestricted use, distribution, and reproduction in any medium, provided the original work is properly cited.

In crude oil production from brown fields or heavy oil, there is production of water in oil emulsions which can either be controlled or avoided. This emulsion resulted in an increase in viscosity which can seriously affect the production of oil from sand phase up to flow line. Failure to separate the oil and water mixture efficiently and effectively could result in problems such as overloading of surface separation equipments, increased cost of pumping wet crude, and corrosion problems. Light hydrocarbon diluent was added in varied proportions to three emulsion samples collected from three different oil fields in Niger delta, Nigeria, to enhance the demulsification of crude oil emulsion. The viscosity, total petroleum hydrocarbon, and quality of water were evaluated. The viscosity of the three emulsions considered reduced by 38,31 , and $18 \%$. It is deduced that the increase in diluent blended with emulsion leads to a corresponding decrease in the value of viscosity. This in turn enhanced the rate of demulsification of the samples. The basic sediment and water (BS\&W) of the top dry oil reduces the trace value the three samples evaluated, and with optimum value of diluent, TPH values show that the water droplets are safe for disposal and for other field uses.

\section{Introduction}

Emulsion is defined as a system in which one liquid is relatively distributed or dispersed, in the form of droplets, in another substantially immiscible liquid. Emulsions have long been of great practical interest due to their widespread occurrence in everyday life which occurs due to reliance of the behaviour of the emulsion on the magnitude and range of the surface interaction. They may be found in important areas such as food, cosmetics, pulp and paper, biological fluids, pharmaceutical, agricultural industry, and petroleum engineering. In production and flow assurance, the two commonly encountered emulsion types are water droplet dispersed in the oil phase and termed as water-in-oil emulsion $(\mathrm{W} / \mathrm{O})$ and if the oil is the dispersed phase, it is termed oilin-water $(\mathrm{O} / \mathrm{W})$ emulsion [1].

Water-in-oil crude oil emulsions may be encountered at all stages in the petroleum production and in processing industry. With presence of water, they are typically undesirable and can result in high pumping costs and pipeline corrosions and increase the cost of transportation [2].
Reduced throughput is needed to introduce special handling equipment, contribute to plugging of gravel pack at the sand phase [3], and affect oil spill cleanup [4].

In their research work, Micheal et al. used bottle test method to simulate field condition of four emulsion samples (two Canadian and two Venezuelan emulsions) in order to determine the variables that affect emulsion stability. They were able to evaluate response to the different emulsion based on bottle test data by introducing thirty-six different demulsifiers to enable them to probe emulsion stability. Linear regression and partition tree analysis were used to analyze the effect of various variables on emulsion stability and were able to conclude that solid content significantly affects emulsion stability. Beside solid content crude oil properties, water chemistry and process condition also influence emulsion stability [5].

Christophe et al. evaluated and compared emulsion formed by different parts of the indigenous amphiphiles (the light, the intermediate, or the heavy ones) to determine their contribution to emulsion stability. The emulsions formed with the light and intermediate fractions separated immediately 
when the agitation stopped. The most stable emulsions were formed with the fraction of crude that distilled at temperature greater than $520^{\circ} \mathrm{C}$, suggesting that the amphiphiles with the highest molecular weight, that is, resins and asphaltenes, play a major role in the protection of water droplet against coalescence [6]. This is consistent with many recent findings that the presence of these components enhanced w/o emulsion stability (Rondón et al. and Ekott and Akpabio [7, 8]). Others factors that affect emulsion stability are fine solids, temperature, size of water droplet, and brine composition [9], which is consistent with the work of previous authors $[5,6]$.

Despite the success of enhanced oil recovery (EOR) process, one of the problems associated with the process is emulsion problem. Efeovbokhan et al. observed that physical factors that enhance oil recovery can also greatly contribute to the formation of very stable emulsions because EOR-induced emulsions are established by surfactant/polymer (SP) and alkaline/surfactant/polymer (ASP) processes which makes breaking of emulsion different from naturally occurring emulsions which are stabilized by asphaltenes and resins [10]. Traditional demulsifiers are often not effective on emulsions created by chemical floods; therefore, the performance of demulsifier in surfactant/polymer-flooding-induced emulsion depends on the selection of the best demulsifier with respect to the system under consideration [11]. In breaking of surfactant/polymer-flooding-induced emulsion with the use of surfactant, Oseghale et al. worked on separation of oilwater emulsions expected during chemical enhanced recovery operations using crude oil from a field in Niger delta during surfactant/polymer flooding operation. Surfactant $N$ octyltrimethyammonium bromide $\left(\mathrm{C}_{8} \mathrm{TAB}\right)$ was used as the demulsifier and a dosage between 200 and 300 ppm was the optimum dose that yielded oil and water phases with oil content reduction from 550 to $70 \mathrm{ppm}$ after $4 \mathrm{~h}$. Microscopy test confirmed that addition of $\mathrm{N}$-octyltrimethyammonium bromide $\left(\mathrm{C}_{8} \mathrm{TAB}\right)$ produced significant coalescence shortly after it was added to the emulsion, which is in agreement with an increase of the oil droplet size in the presence of the demulsifier. Their findings show that this investigation worked with the principles of using cationic surfactants as demulsifier [12].

With various problems encountered with the presence of emulsion in our system, there is need to find ways of controlling existence of emulsion or preventing it from forming in our system. One of the ways of controlling problems encountered by crude oil emulsion is the ability to predict crude oil behaviour both at the sand phase and during production by building a robust predictive model [13]. Emulsion formation or break up either for oil in water or water in oil emulsion can be characterized based on the property and type of crude oil involved in the formation or break up of emulsion which can assist in formulating method of preventing formation of such emulsion [14]. Nuraini et al. selected four groups of demulsifiers which are amine, natural, polyhydric, and alcohol demulsifier groups serving as breaking agents of stable emulsion. Their findings show that amine demulsifier group exhibited the highest efficiency to break the emulsion compared to polyhydric, alcohol, and natural groups and that demulsifier efficiency depends on two-factor solubility of demulsifier either in water or oil and molecular weight of demulsifier [15].

It has been established from the literatures that one of the ways of breaking stable emulsion is introduction of low dose of demulsifiers. For comprehensive methods of breaking emulsion, the work of Hanapi et al. treated that aspect [2]. Micheal et al. used chemical demulsifiers and statistical analysis to classify emulsion. They obtained emulsion from the field and treated the emulsion with thirty-eight chemicals that serve as demulsifiers at nine different sites. The tests were tailored towards determination of water droplet, oil dryness, and oil-water interface which were analyzed using several statistical tools. A correlation was developed for water droplet, oil dryness and oil-water interface. The results show that water droplet significantly affect oil-water interface than oil dryness [16].

Crude oil emulsions are complex and should be characterized as completely as possible. Droplet-size distribution, interfacial phenomena, and the nature of organic and inorganic components are important. The viscosity of the emulsion is affected by both the water content and droplet size distribution $[17,18]$. The increase in aqueous phase of the emulsion leads to an increase in viscosity of emulsion which in turn aggravates flow of emulsion in conduct either at the sand phase or through the surface facilities [3, 19]. Stable waterin-oil emulsions have been generally found to exhibit high interfacial viscosity and/or elasticity modulus. Viscosity of crude oil emulsion was found to increase with increase in water and decreased with increase in speed of rotation of spindle when demulsifier is added [20]. The increase of the interfacial rheological parameters has been attributed to nonNewtonian nature of emulsion [20] and physical cross-links between the asphaltene particles adsorbed at the water-oil interface [21]. Demulsification of emulsion proved to be a good method of breaking emulsions but with an influence of viscosity still unaccounted for in most of the researches; this research will study the effect of adding a diluent to emulsion samples treated with diluent for three different water in oil crude emulsions collected from three different oil fields from three operators in Niger delta, Nigeria.

\section{Materials and Methods}

2.1. Sampling. Fresh crude oil emulsions were collected from the three oil fields flow stations operated by three different operators in the Niger delta in Nigeria, namely, Fields A, B, and $\mathrm{C}$.

At the sampling points in all the three oil field mentioned above, crude oil was collected at both east and west directional sampling pipes. This to ensure that pure emulsion interface is collected and not either gas or water phase. The emulsions are collected in a tightly sealed container. The experiment was carried out after four hours from the time of sampling to avoid ageing of the crude oil. Table 1 show the initial properties of the three water-in-oil emulsion samples used for the experimental work.

Gasoline used as the diluent for this experiment was gotten from the Nigeria National Petroleum Cooperation 
TABLE 1: Properties of crude oil emulsions used for the analysis.

\begin{tabular}{lccc}
\hline & Field A & Field B & Field C \\
\hline Temperature $\left({ }^{\circ} \mathrm{C}\right)$ & 55 & 60 & 50 \\
Production rate $\left(\mathrm{m}^{3} /\right.$ day $)$ & 11,000 & 32,000 & 41,000 \\
Viscosity $\left(\mathrm{mPas}^{-1}\right)$ & 80 & 100 & 215 \\
Residence time $(\mathrm{hrs})$ & 11 & 7 & 15 \\
API gravity $\left({ }^{\circ}\right)$ & 23 & 22 & 21 \\
Water cut $(\%)$ & 51 & 8 & 10 \\
Demulsifier volume used $(\mathrm{ppm})$ & 3 & 18 & 6 \\
\hline
\end{tabular}

(NNPC) Refinery, Port Harcourt, Nigeria. Two types of demulsifiers were used by the operators for breaking of the emulsions formed. Fields A and C used PhaseTreat 4633, while Field B used PhaseTreat 6074.

The equipment used for the analysis are water bath, Checktemp1 digital thermometer, Cannon Fenske viscometer, Model HT 5001-201, six-ounce Pyrex bottles with volume $100 \mathrm{~mL}$, Beaker (100 mL), Socorex Syringe micropipette, Model: Dossy TM 174 premium, Centrifuge Machine: Robinson Centrifuge, Model T.0.2, serial no. T724, Wooden product bottle shaker, and $10 \mathrm{~mL}$ measuring cylinder.

2.2. Experimental Procedure. Each of the crude oil samples was analyzed in different setups; the three crude oil samples were treated according to the properties of the oilfield where they were collected. These properties vary in terms of temperature, rate of chemical injection, nature of process terminal, and time of processing, which will dictate the type of demulsifier chemical to be used. A water bath was set up and maintained at a temperature of $60^{\circ} \mathrm{C}$ equivalent to the average process temperature of the oil fields. This temperature was held constant to neglect the effect of temperature on the viscosity of the crude oil samples.

Six test bottles of capacity $100 \mathrm{~mL}$ were labeled according to their corresponding wells with $\mathrm{A}, \mathrm{B}$, and $\mathrm{C}$, with suffixes 1 to 6 on each of the wells. The suffix 1 denotes $0 \mathrm{~mL}$ of diluent, 2 is $2 \mathrm{~mL}, 3$ is $4 \mathrm{~mL}, 4$ is $6 \mathrm{~mL}, 5$ is $8 \mathrm{~mL}$, and 6 is $10 \mathrm{~mL}$ of diluent. The bottles were filled with crude oil and gasoline to make up a volume of $100 \mathrm{~mL}$. Prior to addition of diluent to the emulsion, demulsifier was added in a ratio of one-third of the amount used by the operators where the samples were collected. The samples (emulsion + gasoline) were placed in a bottle shaker and agitated thoroughly with 50 vertical shakes and 50 horizontal shakes to homogenize the diluent with the continuous phase of the emulsion. The bottle was returned to water bath after blending for ten minutes after which percentage-free water was recorded.

The viscosities reading of various combinations of the blend of demulsifier, emulsion, and diluent were obtained using the Cannon Fenske viscometer according to the procedure recommended by ASTM D445 (Norman) [22].

Basic sediment and water of the emulsion was determined using the method described in the published work of Sunil et al. [9]. Total petroleum hydrocarbon was measured by

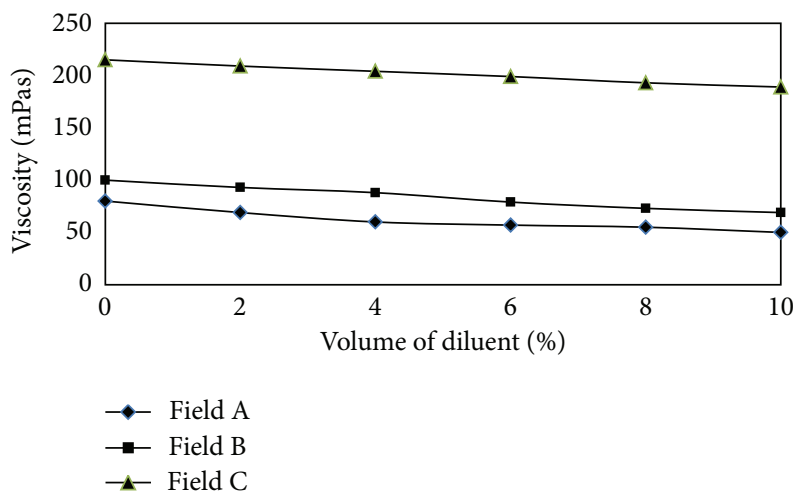

FIGURE 1: Volume of diluent against viscosity of emulsion.

using TPH analyzer (Model HC-404). A sample of the effluent water was taken and fed into this analyzer and the reading recorded in parts per million, ppm.

\section{Results and Discussion}

3.1. Flow Assurance. Effect of diluent on viscosity is illustrated in Figure 1. Gasoline was added to the three crude oil emulsions from $2 \mathrm{~mL}$ to $10 \mathrm{~mL}$ and emulsion volume is reduced from 100 to $90 \mathrm{~mL}$. There is viscosity reduction when diluent is introduced. The reduction in viscosity is proportional to the increase in the volume of gasoline. Effect of viscosity reduction is a function of initial viscosity of the emulsion because from the graph the reductions of viscosity in fields A, B, and $\mathrm{C}$ are 38,31 , and $17 \%$, respectively when $10 \mathrm{~mL}$ of gasoline was added to them and their initial viscosity are 80, 100, and $215 \mathrm{mPas}$ which means that the sample with lowest value of viscosity experienced the highest percentage reduction in viscosity value and the sample with the highest viscosity experienced the lowest percentage reduction in viscosity value.

3.2. Rate of Separation of Water. It was observed from Figures 2 and 3 that introduction of diluent affect Basic sediment and water (BS\&W) of crude oil emulsion. The BS\&W of Field A crude oil emulsion sample was originally $0.5 \%$ when treated with an injection rate of $1 \mathrm{ppm}$, and without blending with diluent. The value reduces as the volume of diluent increases until $8 \mathrm{~mL}$ when the value is zero. There is reduction in the value of BS\&W of samples from fields B and C which was initially at $0.7 \%$ after the addition of $6 \mathrm{~mL}$ and $2 \mathrm{~mL}$ of demulsifiers to them to zero and $0.2 \%$ when the volume of the diluent was increased to $10 \mathrm{~mL}$. Also viscosity of emulsion plays an important role in the analysis of BS\&W because Field A reduces its value of BS\&W to zero when the volume of diluent is $8 \mathrm{~mL}$, B when the volume is $10 \mathrm{~mL}$, and for $\mathrm{C}$ the value is at $0.2 \%$ when the volume of the diluent is at $10 \mathrm{~mL}$. Field A used the lowest amount of diluent because it has the lowest viscosity while $\mathrm{C}$ has the the highest amount of diluent since it has the highest viscosity. Therefore, it is established that the diluent is capable of increasing reduction of BS\&W in crude oil emulsions. 


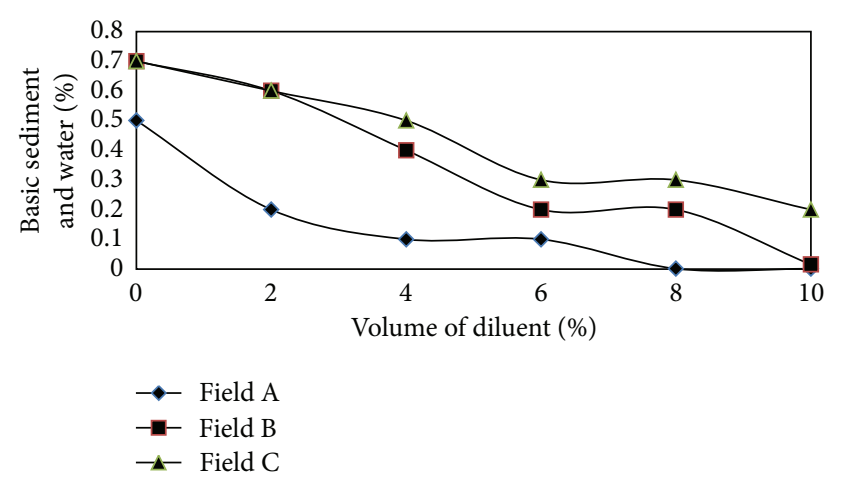

FIgURE 2: Volume of diluent against basic sediment and water.

3.3. Total Petroleum Hydrocarbon. The TPH of each sample initially reduces with the increase in the amount of gasoline added, but later started increasing after a particular blending point. This undesirable effect is believed to be caused by excess gasoline in the mixture finding their way into the aqueous phase.

Field A crude sample maintained a good TPH of $64 \mathrm{ppm}$ which reduces as the diluent value increased between 0 and $2 \mathrm{~mL}$, but there is a sharp increase in the value of TPH as the volume of diluent is increased above $2.2 \mathrm{~mL}$. These reduction and rising of the TPH value with the increase in the diluent volume are attributed to the relative tightness of the crude oil emulsion. Tightness is the degree at which the water droplets are held in suspension and resist separation.

Fields B and C crude emulsions demonstrated high TPH values of $84 \mathrm{ppm}$ and $93 \mathrm{ppm}$. Initially, blending showed less effect of diluent on the TPH of these two crudes between 0 and $2 \mathrm{~mL}$. The TPH values of $\mathrm{B}$ and $\mathrm{C}$ reduced to $60 \mathrm{ppm}$ at $4 \mathrm{~mL}$ of diluent, which was constant till $6 \mathrm{~mL}$ of diluent. Above $6 \mathrm{~mL}$ of diluent, there is an increase in the value of TPH fields of B and C to 70 when the volume of diluent is $10 \mathrm{~mL}$. Apart from tightness of the emulsion, excess diluent can penetrate aqueous phase of the emulsion which will increase the value of its TPH.

3.4. Bottle Test. The demulsification bottle test was carried out and results on water droplet are taken after 5, 20, 30, 60, and 720 minutes. Water droplet is the separation of water from the surface of emulsion formed. The effect of addition of diluent on each crude oil sample was monitored on the rate of water droplet from each of the emulsion samples. The suffixes after the fields denotations $\mathrm{A}, \mathrm{B}$, and $\mathrm{C}$ indicated the variation of diluent concentration added to the emulsion samples which read $1,2,3,4,5$, and 6 for $0,2,4,6,8$, and $10 \mathrm{~mL}$ of diluent concentration.

From Figure 4, depending on the amount of diluent blended with the emulsion there was a corresponding increase in the rate of water droplet with time. When $2 \mathrm{~mL}$ of diluent was blended with emulsion, there was no water droplet after 5 minutes; it increased to $6 \%$ at 20 minutes and $22 \%$ at the end of 60 minutes after which there was no further droplet till the end of 720 minutes which was illustrated in A1 in Figure 4. In A-2, the trend was similar to that of A-1 but

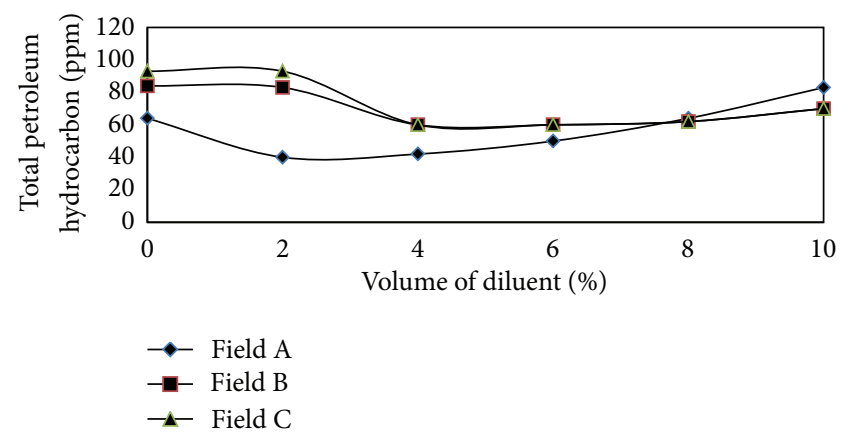

Figure 3: Volume of diluent against basic sediment and water.

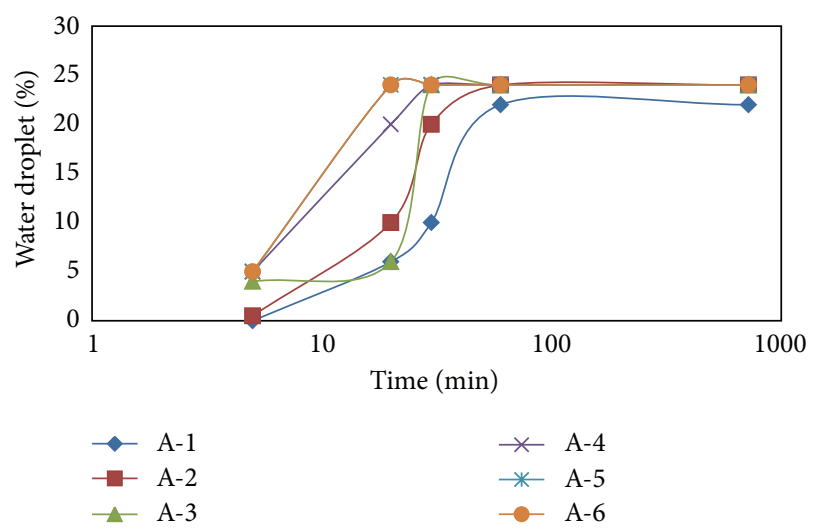

FIgURE 4: Water droplet against time at various emulsion/diluent ratio for Field A.

the final water droplet value is $24 \%$. In A-3 and A-4, there was a water droplet of 4 and $5 \%$ at 5 minutes which increased to 6 and $20 \%$ after 20 minutes, $24 \%$ at the end of 30 minutes after which there was no further droplet till the end of 720 minutes.

In A-5 and A-6, water droplet was $5 \%$ at 5 minutes, $24 \%$ at the end of 20 minutes, and remained constant till the end of 720 minutes. Generally, it was observed that low amount of diluent take longer time for water to drop from the emulsion but as the volume of diluent increased, the time required for water to drop out of the emulsion decreased.

Figure 5 shows the behavior of change in diluent concentration with rate of water droplet for emulsion samples collected from Field B. When no diluent was blended with the emulsion samples obtained from Field B, there was no droplet of water until after 60 minutes with a value of $4 \%$ and progressively increased to $6 \%$ at the end of 720 minutes. In B2 to B-4, the trend of the curve followed the trend experienced in B-1 only that the rate of water droplet was faster and higher than that of B-1 with a value of 8,10 , and $10 \%$, respectively, for B-2, B-3, and B-4 at the end of 720 minutes. In B-5 and B-6, water droplet was experienced earlier than the previous four situations with droplet of 3 and $4 \%$ at 5 minutes; it increased to 5 and $10 \%$ at 20 minutes and was $12 \%$ from 30 to 720 minutes when the analysis was terminated.

When no diluent was blended with the emulsion obtained from Field C and when $2 \mathrm{~mL}$ is blended with it, the behavior of their chart was similar and illustrated in C-1 and C-2 in 
TABLE 2: Effect of diluent on water quality and interface for emulsions after 720 minutes.

\begin{tabular}{|c|c|c|c|c|c|c|c|}
\hline \multirow{2}{*}{ Volume of emulsion } & \multirow{2}{*}{ Volume of diluent } & \multicolumn{2}{|c|}{ Field A } & \multicolumn{2}{|c|}{ Field B } & \multicolumn{2}{|c|}{ Field C } \\
\hline & & Water quality & Interface & Water quality & Interface & Water quality & Interface \\
\hline 100 & 0 & Dirty & Cloudy & Dirty & Cloudy & Dirty & Cloudy \\
\hline 98 & 2 & Fair & Stained & Dirty & Stained & Dirty & Cloudy \\
\hline 96 & 4 & Fair & Sharp & Fair & Stained & Dirty & Stained \\
\hline 94 & 6 & Clean & Sharp & Fair & Sharp & Fair & Sharp \\
\hline 92 & 8 & Clean & Sharp & Clean & Sharp & Clean & Sharp \\
\hline 90 & 10 & Clean & Sharp & Clean & Sharp & Clean & Sharp \\
\hline
\end{tabular}

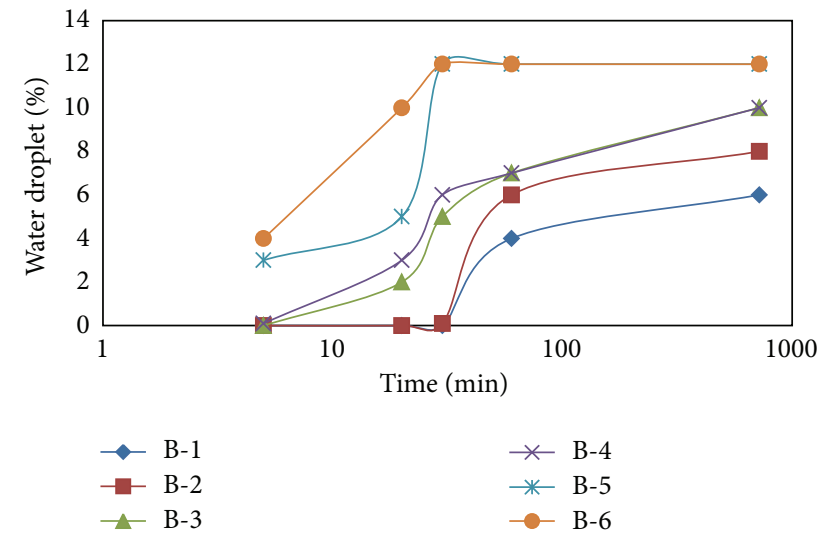

FIGURE 5: Water droplet against time at various emulsion/diluent ratio for Field B.

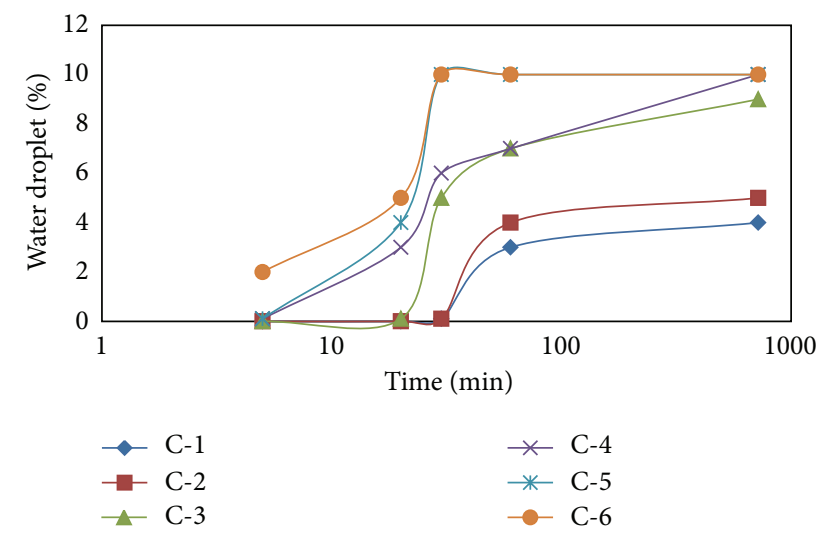

Figure 6: Water droplet against time at various emulsion/diluent ratio for Field C.

Figure 6. There was no water droplet in the two charts until at 30 minutes with water droplet value of $0.1 \%$ which increased to 4 and $5 \%$ at 720 minutes. C-3 and C- 4 followed the trend observed in C-1 and C-2 only that water droplet rate was faster with a value higher than that of $\mathrm{C}-1$ and $\mathrm{C}-2$. The value of water droplet at 720 minutes in C-3 and C-4 are 9 and $10 \%$. In C-5 and B-6, water droplet was experienced earlier than the first four situation. After 5 minutes, water droplet was 1 and $2 \%$ which increased to 4 and $5 \%$ at 20 minutes. The value of water droplet remains constant at 30 till 720 minutes at $10 \%$.
3.5. Water Quality. The quality of water droplet and observation at the oil and water interface after separation for the three crude oil emulsions are captured in Table 2. For the three crude oil emulsion samples when diluent is not blended with the emulsion, the water quality is dirty and the interface between the water droplet and oil phase is cloudy after 720 minutes. As the diluent volume blended with emulsion increased there, is an improvement in the quality of water change from dirty to clean (i.e., there is no residual emulsion or oil in the water) and the interface between oil and dropped water changes from stained to sharp (i.e., there is a distinct different between water phase and oil phase).

\section{Conclusion}

Generalized conclusions are hence drawn from the observation of the three samples of crude oil used for this bottle test as follows:

(i) the viscosity of the three water-in-crude oil emulsions considered is inversely proportional to the increase in volume of diluent (gasoline) blended with emulsion. Also the effect of gasoline on the viscosity reduction was observed to be a function of the heaviness of the crude oil emulsion because the higher the viscosity of the emulsion the lower the reduction percentage in its viscosity value. Blending of emulsion reduced the viscosity of the three samples considered by 38,31 , and $17 \%$, respectively.

(ii) Basic sediment and water (BS\&W) reduces as the volume of diluent blended with the emulsion increases. This is also a function of viscosity of emulsion prior to blending because BS\&W decreased with the decrease in the value of viscosity.

(iii) Total petroleum hydrocarbon (TPH) decreases with the increase in the volume of diluent until optimum concentration of diluent is reached and the TPH increases with further increase in volume of diluent. Optimum value varied for the three crude oil emulsions considered in the analysis. However, above optimum volume of diluent the TPH of the effluent increases which creates another problem when it comes to water disposal.

\section{References}

[1] D. Langevin, S. Poteau, I. Hénaut, and J. F. Argillier, "Crude oil emulsion properties and their application to heavy oil 
transportation," Oil and Gas Science and Technology, vol. 59, no. 5, pp. 511-521, 2004.

[2] M. Hanapi, S. Ariffin, A. Aizan, and I. R. Siti, "Study on demulsifier formulation for treating malaysian crude oil emulsion," Tech. Rep., Department of Chemical Engineering, Universiti Technologi Malaysia, 2006.

[3] R. Espinoza and W. Kleinitz, "The impact of Hidden Emulsion on Oil Prooducing wells-stimulation concept and field result," in Proceedings of the SPE European Formation Damage, The Hague, The Netherlands, 2003, SPE paper 00082252.

[4] F. Merv and F. Ben, "Studies of the formation process of waterin-oil emulsions," Marine Pollution Bulletin, vol. 47, no. 9-12, pp. 369-396, 2003.

[5] K. P. Micheal, C. Shaokum, and C. M. Samuel, "The key to Predicting Emulsion stability: solid content," in Proceedings of the SPE International Symposium on Oil Field Chemistry, Houston, Tex, USA, 2005, SPE paper 93008.

[6] D. Christophe, A. David, S. Anne, G. Alain, and B. Patrick, "Stability of water/crude oil emulsions based on interfacial dilatational rheology," Journal of Colloid and Interface Science, vol. 297, no. 2, pp. 785-791, 2006.

[7] M. Rondón, J. C. Pereira, P. Bouriat, A. Graciaa, J. Lachaise, and J. L. Salager, "Breaking of water-in-crude-oil emulsions. 2. Influence of asphaltene concentration and diluent nature on demulsifier action," Energy and Fuels, vol. 22, no. 2, pp. 702-707, 2008.

[8] E. J. Ekott and E. J. Akpabio, "Influence of asphaltene content on demulsifiers performance in crude oil emulsions," Journal of Engineering and Applied Sciences, vol. 6, no. 3, pp. 200-204, 2011.

[9] K. Sunil, A. Abdullah, and N. S. Meeranpillal, "An Investigative study of potential emulsion problems before field development," in Proceedings of the SPE Annual Technical Conference and Exhibition, San Antonio, Tex, USA, 2007, SPE paper 102856.

[10] V. Efeovbokhan, T. Akinola, and F. Hymore, "Performance evaluation of formulated and commercially available de-emulsifiers," in Nigerian Society of Chemical Engineers Proceedings (NSChE '10), vol. 40, pp. 87-99, 2010.

[11] D. T. Nguyen and N. Sadeghi, "Selection of the right demulsifier for chemical enhanced oil recovery," in International Symposium on Oilfield Chemistry, The Woodlands, Tex, USA, April 2011.

[12] C. I. Oseghale, E. J. Akpabio, and G. Udottong, "Breaking of oilwater emulsion for the improvement of oil recovery operations in the Niger Delta Oilfields," International Journal of Engineering and Technology, vol. 2, no. 11, pp. 1-7, 2012.

[13] B. Fu, "Flow assurance-a technological review of Managing fluid behaviour and solid deposition to Ensure optimum flow," in Proceedings of the 7th Annual International Forum for deepwater Technologies (Deeptec '00), Aberdeen, UK, January 2000.

[14] C. Noïk, H. Malot, C. Dalmazzone, and A. Mouret, "Encapsulation of crude oil emulsions," Oil and Gas Science and Technology, vol. 59, no. 5, pp. 535-546, 2004.

[15] M. Nuraini, H. N. Abdurahman, and A. M. S. Kholijah, "Effect of chemical breaking agents on water-in crude oil emulsion system," International Journal of Chemical and Environmental Engineering, vol. 2, no. 4, pp. 1-5, 2011.

[16] K. P. Micheal, C. Shaokum, A. M. Robert, and C. M. Samuel, "Classifying crude oil emulsion using chemical demulsifiers and stastical analyses," in Proceedings of the SPE Annual Technical Conference and Exhibition, Denver, Colo, USA, 2003, SPE paper 84610 .
[17] S. D. Taylor, "Investigations into the Electrical and rheological Behaviour of W/O-emulsions in high voltage Gradients," Colloid \& Surfaces, vol. 29, pp. 25-51, 1988.

[18] D. G. Thompson, A. S. Taylor, and D. E. Graham, "Emulsification and demulsification related to crude oil production," Colloid \& Surfaces, vol. 15, pp. 175-189, 1987.

[19] T. J. Jones, E. L. Neustadter, and K. P. Whittingham, "Waterin-crude oil emulsion stability and emulsion destabilization by chemical demulsifiers," Journal of Canadian Petroleum Technology, vol. 17, no. 2, pp. 100-108, 1978.

[20] N. H. Abdurahman and W. K. Mahmood, "Stability of water-incrude oil emulsions: effect of cocamide diethanolamine (DEA) and Span 83," International Journal of Physical Sciences, vol. 7, no. 41, pp. 5585-5597, 2012.

[21] J. D. McLean and P. K. Kilpatrick, "Effects of asphaltene solvency on stability of water-in-crude-oil emulsions," Journal of Colloid and Interface Science, vol. 189, no. 2, pp. 242-253, 1997.

[22] J. H. Norman, Non-Technical Guide to Petroleum, Geology, Exploration, Drilling and Production, Penswell Corporation, Tulsa, Okla, USA, 2nd edition, 2001. 

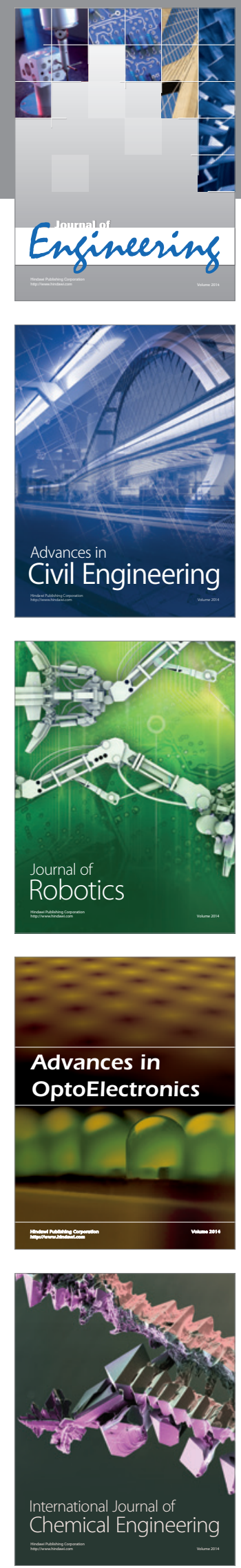

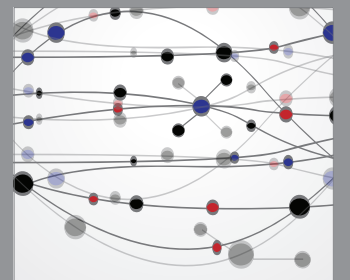

The Scientific World Journal
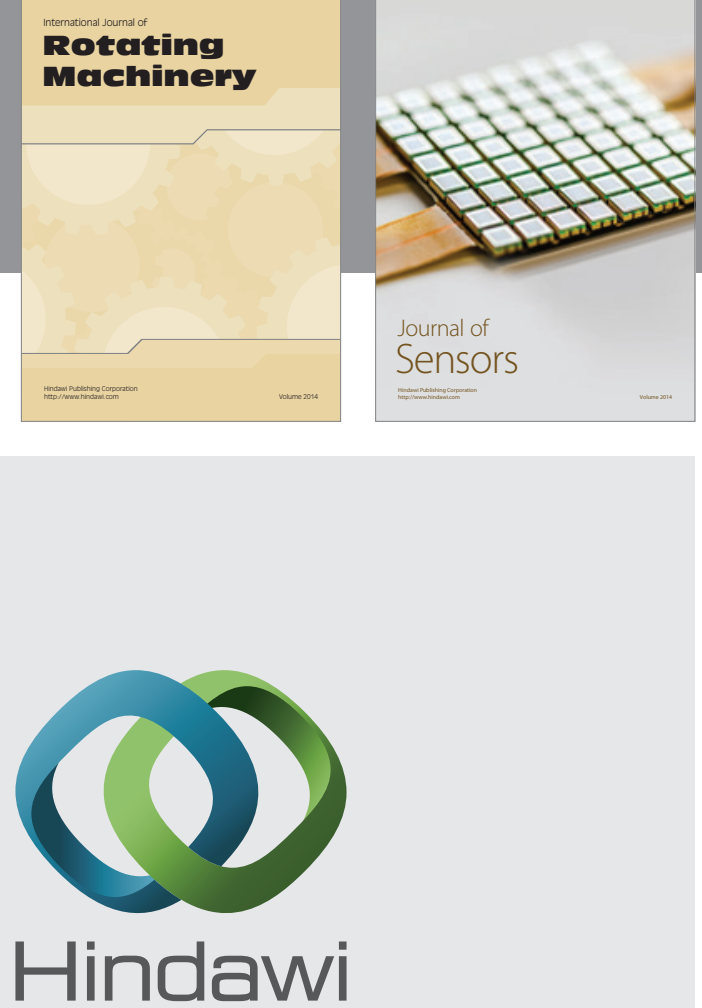

Submit your manuscripts at http://www.hindawi.com
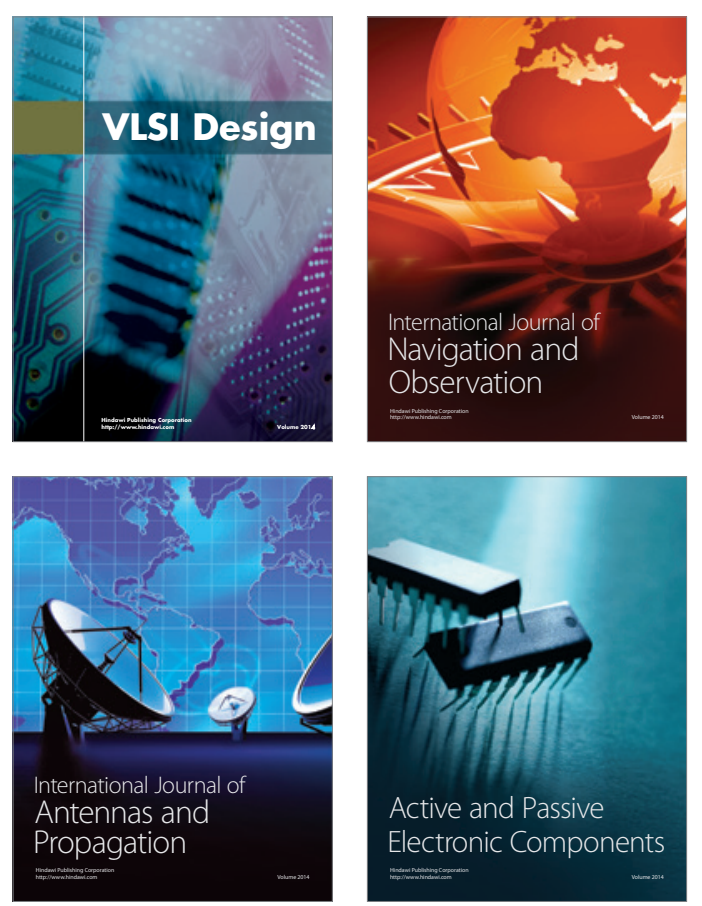
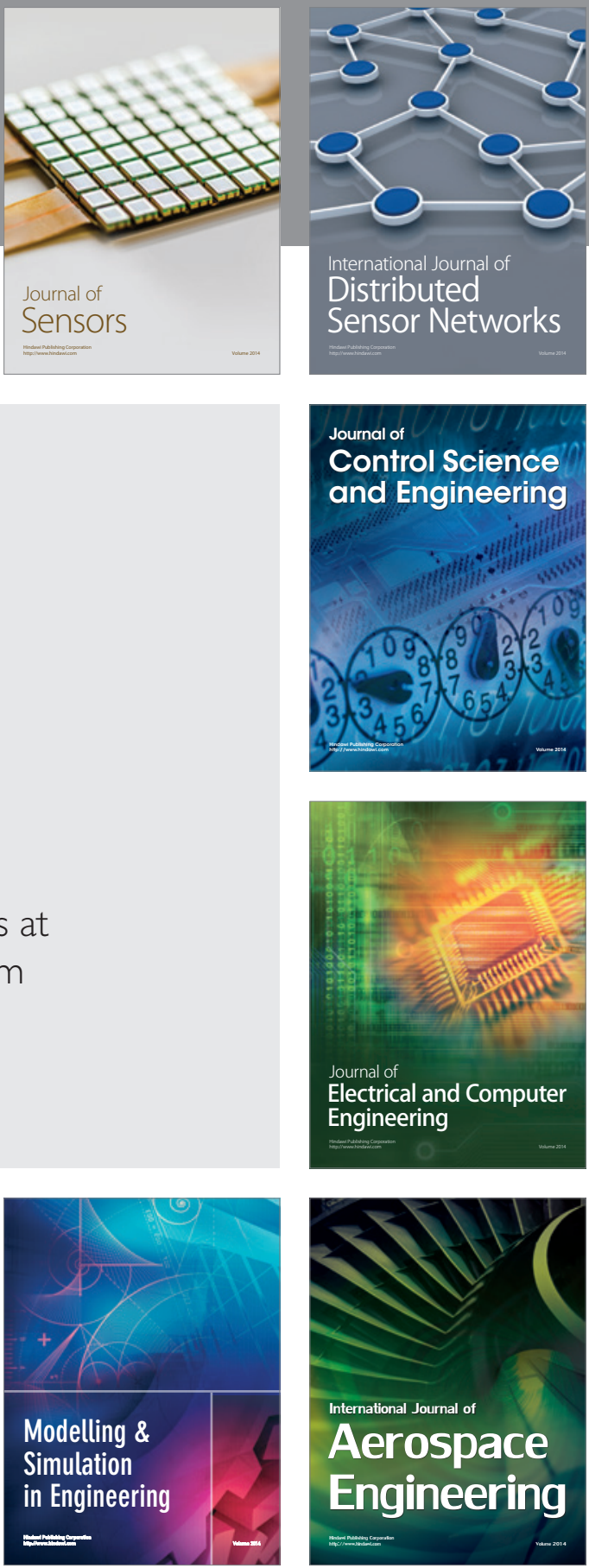

Journal of

Control Science

and Engineering
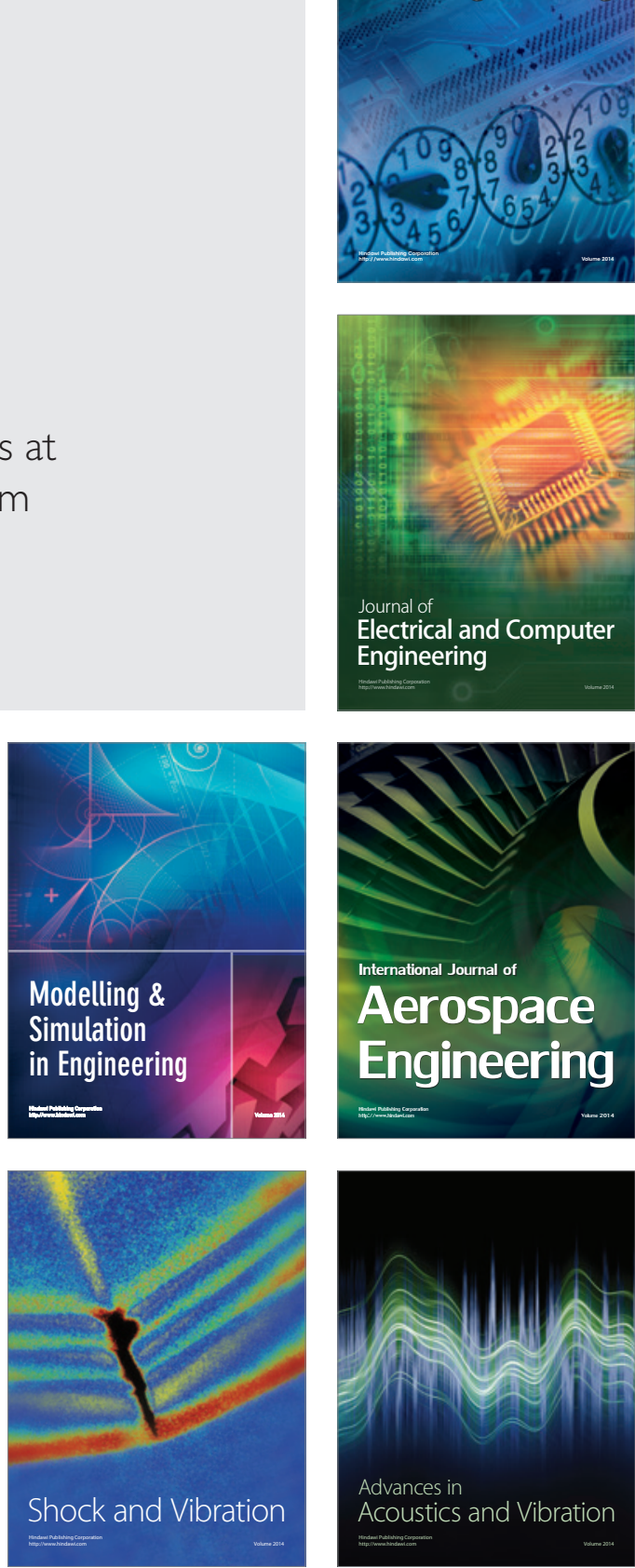